\title{
openheart Is concomitant aspirin helping novel oral anticoagulants? Focus on apixaban
}

James J DiNicolantonio, ${ }^{1}$ Ales Tomek, ${ }^{2}$ Pascal Meier, ${ }^{3,4}{ }^{4}$ James H O'Keefe, ${ }^{1}$
Fabrizio D'Ascenzo, ${ }^{5}$ Enrico Cerrato, ${ }^{5}$ Saurav Chatterjee, ${ }^{6}$ Giuseppe Biondi-Zoccai ${ }^{7}$

To cite: DiNicolantonio JJ, Tomek A, Meier P, et al. Is concomitant aspirin helping novel oral anticoagulants? Focus on apixaban. Open Heart 2014;1:e000134. doi:10.1136/openhrt-2014000134

Accepted 1 September 2014

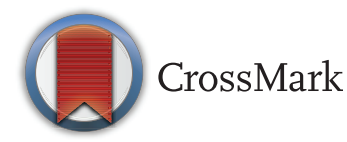

${ }^{1}$ Mid America Heart Institute, St. Luke's Hospital, Kansas City, Missouri, USA

${ }^{2}$ Department of Neurology, Charles University in Prague, 2nd Faculty of Medicine, University Hospital Motol, Prague, Czech Republic

${ }^{3}$ The Heart Hospital, University College London Hospitals UCLH, London, UK ${ }^{4}$ Yale Medical School, New Haven, Connecticut, USA ${ }^{5}$ Division of Cardiology, University of Turin, Città Della Salute e Della Scienza,

Torino, Italy

${ }^{6}$ St Luke's Roosevelt Hospital Center, New York, New York, USA

${ }^{7}$ Department of Medico-

Surgical Sciences and Biotechnologies, Sapienza University of Rome, Latina, Italy

Correspondence to Dr James J DiNicolantonio; jjdinicol@gmail.com

\section{INTRODUCTION}

Apixaban is the third novel oral anticoagulant approved by the Food and Drug Administration for the reduction in the risk of stroke in patients with atrial fibrillation. Apixaban showed a significant reduction in mortality as well as a reduction in strokes compared with warfarin in the Apixaban for Reduction in Stroke and Other Thromboembolic Events in Atrial Fibrillation (ARISTOTLE) trial. ${ }^{1}$ However, it is unclear how much of this 'benefit' was derived from the negative benefit to risk ratio (RR) when combining aspirin with warfarin compared with warfarin alone. Approximately one-third of patients received combination warfarinacetylsalicylic acid (ASA) therapy in ARISTOTLE at baseline $(31.3 \%$ on apixaban, $30.5 \%$ on warfarin), with approximately 20$25 \%$ of patients receiving aspirin with longterm anticoagulation, ${ }^{2}$ despite the fact that only $14 \%$ of patients randomised to warfarin had a definitive indication for concomitant aspirin therapy (ie, patients having a previous myocardial infarction (MI)). Most patients receiving aspirin had arterial vascular disease, but the majority did not have a recent MI, which is a more appropriate indication for concomitant warfarin-ASA therapy (ie, a history of MI $<6$ months, depending on the stent used). The high percentage of warfarin-ASA use in ARISTOTLE, despite no clear indication for many of these individuals, introduces a significant confounder. Did aspirin increase the harm in patients on warfarin over and above what would be seen in patients receiving apixaban?

\section{ARISTOTLE}

A total of $20-25 \%$ of patients were on combination warfarin-ASA in the ARISTOTLE trial, despite the fact that only $14 \%$ and $16 \%$ of patients had a history or occurrence of MI before and throughout the trial duration, respectively. ${ }^{1}{ }^{2}$ It is a conundrum as to why there was such a high rate of concomitant
warfarin-ASA allowed, when a favourable benefit to risk ratio for combination warfarin-ASA therapy has only been shown for patients with mechanical heart valves, the very patients excluded from this trial. ${ }^{134}$

Combined warfarin-ASA therapy confers a $1-2 \%$ absolute risk increase in major bleeds per year compared with warfarin alone, with each major bleed conferring a 9-10\% death rate per year. ${ }^{3-5}$ Therefore, for every 1000 patients treated with combination warfarin-ASA in ARISTOTLE, 20 additional major bleeds and 2 additional deaths per year would occur compared with the group on warfarin alone. It has been previously shown that aspirin in addition to another new oral anticoagulant (dabigatran $150 \mathrm{mg}$ twice daily) does not significantly increase major bleeds. Indeed, aspirin did not significantly increase major bleeds, clinically relevant plus major bleeds, or total bleeds, compared with dabigatran $150 \mathrm{mg}$ twice daily without aspirin in the Dabigatran With or Without Concomitant Aspirin Compared With Warfarin Alone in Patients With Nonvalvular Atrial Fibrillation (PETRO) study. ${ }^{6}$ This is despite the fact that dabigatran increased gastrointestinal bleeding compared with warfarin in the RE-LY trial. Thus, aspirin may not increase bleeding with concomitant use of apixaban (as it did not with dabigatran); it is generally recognised that apixaban may be the safest novel oral anticoagulant compared to dabigatran and rivaroxaban. If indeed aspirin does not increase the risk of a major bleed with apixaban, then this could potentially affect the interpretation of the ARISTOTLE trial. ${ }^{7}$

Further evidence supporting the hypothesis that concomitant aspirin use with warfarin may be partially driving apixaban's 'benefit' is the fact that the primary efficacy outcome, stroke and systemic embolism were significantly improved with apixaban versus warfarin in those who were taking aspirin at randomisation (70 events $(1.3 \%$ per year) vs 94 events (1.9\% per year), OR=0.72, $95 \%$ CI 0.53 to $0.99, \mathrm{p}=0.0474)$, whereas there was no 
significant benefit with apixaban versus warfarin in patients who were not taking aspirin at randomisation (142 events (1.2\% per year) vs 171 events ( $1.5 \%$ per year), $\mathrm{OR}=0.84,95 \%$ CI 0.67 to $1.05, \mathrm{p}=0.1226) .{ }^{1}$ Additionally, these data indicate that for warfarin patients treated with aspirin at baseline, stroke or systemic embolism was worse (1.9\% per year) compared with warfarin patients not treated with aspirin at baseline (1.5\% per year; RR 1.23 ; $95 \%$ CI 0.96 to $1.57, p=0.11$, albeit not significantly different), whereas stroke/systemic embolism for apixaban patients treated with aspirin at baseline $(1.3 \%)$ was very similar to apixaban patients not taking aspirin at baseline (1.2\%; RR 1.10 ; $95 \%$ CI 0.83 to $1.46, p=0.51)$. While these data should be interpreted with caution, as patients receiving vs those who are not receiving aspirin are no longer randomised (ie, patients not receiving aspirin may be at a lower risk and differ in other important ways (hidden confounding) that could be contributing to the difference), it does introduce the possibility that concomitant aspirin use affected patients on warfarin differently (and perhaps more detrimentally) than those on apixaban. Generally, when one medication is superior to another there is a trend for benefit throughout all regions. However, the largest geographic region (Europe, $n=7343$ ) showed no significant benefit with apixaban versus warfarin for reducing stroke and systemic embolism. In summary, the high use of concomitant aspirin with warfarin in ARISTOTLE may have driven some of apixaban's 'benefit' over warfarin (ie, increased harm, especially haemorrhagic stroke).

\section{AVERROES}

The goal of the Apixaban versus Acetylsalicylic Acid to Prevent Strokes (AVERROES) trial was to determine if apixaban is a better choice compared with aspirin for the prevention of stroke in patients with atrial fibrillation. However, a closer look at the dose of aspirin used in this trial seems to question the validity of the results. From the Stroke Prevention in Atrial Fibrillation (SPAF-1) trial, $325 \mathrm{mg}$ of aspirin was the only dose that has been shown to significantly reduce stroke in patients with atrial fibrillation. ${ }^{8}$ However, only $7 \%$ of patients received a $325 \mathrm{mg}$ dose of aspirin in AVERROES, allowing the other 93\% of patients to receive a non-evidence-based dose of aspirin (ie, $<325 \mathrm{mg}$ of aspirin). ${ }^{10}$ Thus, there is no concrete evidence to indicate that apixaban is better at reducing the risk of stroke in patients with atrial fibrillation against an evidence-based dose of aspirin (ie, $325 \mathrm{mg}$ ). As apixaban has recently been approved in Europe and the USA, how is a clinician to decide if an evidence-based dose of aspirin should be used (325 mg) or the more expensive and seemingly more beneficial apixaban? Perhaps it should be the duty of the physicians to explain to their patient that apixaban has not been proven to be superior to an evidence-based dose of aspirin (325 mg) and let the patient decide if it is worth the extra cost.

\section{CONCLUSION}

The above data raise some concern as to whether allowing the concomitant use of aspirin had any effect on the results of ARISTOTLE and AVERROES. It is still uncertain as to why $93 \%$ of the patients in AVERROES received a non-evidence-based dose of aspirin $(<325 \mathrm{mg})$ and why so many patients received a combination of warfarin-ASA therapy (20-25\%) in ARISTOTLE, despite no clear indication in many of them. While these data are considered 'hypothesisgenerating', they should neither be dismissed nor overinterpreted, and perhaps when a clinician is deciding on which oral anticoagulant to use, concomitant aspirin use may (and perhaps should) be a deciding factor.

Contributors JJD wrote the initial draft. AT, PM, JHO, FD'A, EC, SC and GB-Z provided suggestions, edits and read and approved the final draft.

Competing interests GB-Z has consulted or lectured for Astra Zeneca, Bristol Myers Squibb, Pfizer and Sanofi Aventis. AT has consulted and lectured for Pfizer, Boehringer Ingelheim, Bristol-Myers Squibb, Sanofi Aventis and Bayer.

Provenance and peer review Commissioned; internally peer reviewed.

Open Access This is an Open Access article distributed in accordance with the Creative Commons Attribution Non Commercial (CC BY-NC 4.0) license, which permits others to distribute, remix, adapt, build upon this work noncommercially, and license their derivative works on different terms, provided the original work is properly cited and the use is non-commercial. See: http:// creativecommons.org/licenses/by-nc/4.0/

\section{REFERENCES}

1. Granger CB, Alexander JH, McMurray JJ, et al.; ARISTOTLE Committees and Investigators. Apixaban versus warfarin in patients with atrial fibrillation. N Engl J Med 2011;365:981-92.

2. Alexander JH, Lopes RD, Thomas L, et al. Apixaban vs. warfarin with concomitant aspirin in patients with atrial fibrillation: insights from the ARISTOTLE trial. Eur Heart J 2014;35:224-32.

3. Linkins LA, Choi P, Douketis JD. Clinical impact of Bleeding in patients taking oral anticoagulation therapy for venous thromboembolism: a meta analysis. Ann Intern Med 2003;139:893-900.

4. Carrier M, Le Gal G, Wells PS, et al. Systematic review: case-fatality rates of recurrent venous thromboembolism and major bleeding events among patients treated for venous thromboembolism. Ann Intern Med 2010;152:578-89.

5. Douketis JD. Combination warfarin-ASA therapy: which patients should receive it, which patients should not, and why? Thromb Res 2011;127:513-17

6. Ezekowitz MD, Reilly PA, Nehmiz G, et al. Dabigatran with or without concomitant aspirin compared with warfarin alone in patients with nonvalvular atrial fibrillation (PETRO Study). Am J Cardiol 2007;100:1419-26.

7. DiNicolantonio JJ. Dabigatran or warfarin for the prevention of stroke in atrial fibrillation? A closer look at the RE-LY trial. Expert Opin Pharmacother 2012;13:1-11.

8. Ansara AJ, Nisly SA, Arif SA, et al. Aspirin dosing for the prevention and treatment of ischemic stroke: an indication-specific review of the literature. Ann Pharmacother 2010;44:851-62.

9. [No authors listed]. Stroke prevention in atrial fibrillation study. Circulation 1991;84:527-39.

10. Connolly SJ, Eikelboom J, Joyner C, et al.; AVERROES Steering Committee and investigators. Apixaban in patients with atrial fibrillation. N Engl J Med 2011;364:806-17. 


\section{Correction}

DiNicolantonio JJ, Tomek A, Meier P, et al. Is concomitant aspirin helping novel oral anticoagulants? Focus on apixaban. Open Heart 2014;1:e000134. Competing interest section was published with an incomplete sentence. The correct sentence should be 'GB-Z has consulted, lectured and served on the advisory board for Astra Zeneca, Bayer, Bristol Myers Squibb, Pfizer and Sanofi Aventis'.

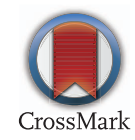

Open Heart 2014;0:e000134corr1. doi:10.1136/openhrt-2014-000134corr1 